\title{
A (des)construção social do diagnóstico de autismo no contexto das políticas de cotas para pessoas com deficiência no mercado de trabalho
}

The social (de)construction of the autism diagnosis in the context of quota policies for people with disabilities in the labour market

\section{Valéria Aydos}

\section{(2) OpenEdition Journals}

\section{Edição electrónica}

URL: http://journals.openedition.org/aa/3492

DOI: $10.4000 /$ aa.3492

ISSN: 2357-738X

\section{Editora}

Programa de Pós-Graduação em Antropologia Social (UnB)

\section{Edição impressa}

Data de publição: 1 junho 2019

Paginação: 93-116

ISSN: 0102-4302

\section{Refêrencia eletrónica}

Valéria Aydos, «A (des)construção social do diagnóstico de autismo no contexto das políticas de cotas para pessoas com deficiência no mercado de trabalho», Anuário Antropológico [Online], v.44 n.1 | 2019, posto online no dia 06 julho 2019, consultado o 28 abril 2021. URL: http:// journals.openedition.org/aa/3492 ; DOI: https://doi.org/10.4000/aa.3492

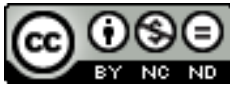

Anuário Antropológico is licensed under a Creative Commons Atribuição-Uso Não-Comercial-Proibição de realização de Obras Derivadas 4.0 International. 


\title{
A (des)construção social do diagnóstico de autismo no contexto das políticas de cotas para pessoas com deficiência no mercado de trabalho ${ }^{1}$
}

\author{
Valéria Aydos \\ Universidade Federal do Rio Grande do Sul - Brasil
}

\section{Introdução}

O cenário de uma crescente propagação midiática sobre o autismo tem trazido à tona um debate acadêmico acerca da produção clínica desse diagnóstico e de uma possível "epidemia de autismo". Tal debate pauta uma problematização sobre a flexibilidade e a complexidade da sua produção como um "diagnóstico clínico" e situado em um espectro altamente diversificado, assim como sobre essa condição ser considerada uma deficiência ${ }^{2}$ ou uma neurodiversidade, uma "maneira diversa de ser e estar no mundo".

Segundo especialistas psi e neurologistas, há hoje um certo consenso ${ }^{3}$ de que o autismo seria "um transtorno do desenvolvimento que prejudica a interação social, compromete a comunicação verbal e não verbal e apresenta padrões repetitivos de comportamento" (White, 2013:114 apud Hollin, 2014a). Na literatura das ciências humanas sobre o tema (Hacking, 2006; Eyal et al., 2010; Ochs et al., 2004; Holin, 2014), há indicações de que o autismo está se tornando cada vez mais presente e se manifestaria (colocando em xeque) exatamente naquilo que mais precisaríamos para "conviver bem em sociedade": as habilidades de socialização e comunicação.

É com o intuito de compreender estas questões que proponho aqui uma análise das especificidades do processo diagnóstico do autismo (Silverman, 2008; Grinker, 2010; Hacking, 2006; Campoy, 2015; Valtellina, 2018) e das disputas em torno do seu status nosológico ${ }^{4}$ (Hacking, 2007; 2009; Ortega et al., 2013) em um contexto específico de interação social: o cotidiano laboral de pessoas com esta condição, espaço no qual pouco se discute e se pesquisa sobre esses temas.

Para tanto, trago ao longo do texto cenas de uma etnografia realizada, entre 2013 e 2017, com dois jovens que tiveram suas primeiras experiências de emprego, a partir de um projeto ${ }^{5}$ de implementação da política de cotas para pessoas com deficiência $^{6}$ no Rio Grande do Sul: Tomás, um jovem de 21 anos, de classe popular, que foi contratado como assistente administrativo em uma empresa de serviços, e 
Ricardo, um rapaz de 26 anos, pertencente às camadas médias, que ingressou como tradutor de inglês-português no setor de comunicação em uma empresa multinacional com sede em Porto Alegre. Na construção de meus argumentos, refiro-me também a relatos de experiências laborais de outros jovens ${ }^{7}$ com autismo, ao acompanhamento do trabalho de assessoria de inclusão da psicopedagoga Clara ${ }^{8}$ e a entrevistas com neurologistas e psiquiatras especializados em autismo.

Tal material contribui para a compreensão do que está em jogo não apenas na coprodução ${ }^{9}$ da categoria autismo na prática cotidiana de diversos atores, mas também, para a identificação das barreiras presentes nas interações sociais dessas pessoas, as quais dificultam ou impossibilitam a efetivação das políticas de inclusão social de pessoas com esta condição no mundo do trabalho. $\mathrm{O}$ debate sobre a produção do diagnóstico de autismo na prática se torna fundamental neste contexto por ser este laudo/atestado identificado com um $\mathrm{CID}^{10}$, legalmente reconhecido como "deficiência”, um pré-requisito para o acesso às cotas.

Parafraseando o termo "categorias perigosas" de Rosenberg (2002), cuja definição seria "aquelas que possuem status ontológico disputado", minha proposta analítica é pensarmos o autismo, visto pelos especialistas como um diagnóstico obscuro, fluido e dinâmico (Hacking, 2006; Eyal et al., 2010; Ochs et al., 2004; Holin, 2014), como produtor de "subjetividades perigosas" e de novas realidades e sensibilidades sociais, cujos efeitos atuam na produção dos sujeitos assim classificados e transformam também a própria política.

\section{Uma epidemia de autismo?}

O debate em torno do autismo tem conquistado crescente visibilidade na mídia e na cena pública nos últimos anos. Logo após a promulgação da Lei Berenice Pianna (Lei 12764, de 2012), a qual "reconhece o autismo como deficiência para todos os fins legais", a revista Veja teve o tema estampado como destaque de capa. Já a Rede Globo de Televisão, lançou uma série no programa Fantástico e abordou o tema com destaque na novela Amor à Vida (ambos em 2013).

Também nas redes acadêmicas e na agenda pública, o autismo parece ser o "diagnóstico do momento" (Fitzgerald, 2015; Ortega et al., 2013). Só nos dois primeiros anos da minha pesquisa, 2013 e 2014, participei de mais de 10 palestras (presenciais e on-line) e de dois grandes congressos científicos sobre o tema; além de recentemente o autismo ter se tornado uma "prioridade na agenda global de saúde mental" (Ortega, 2018). 
Esta maior visibilidade do autismo na cena pública é de fato acompanhada do registro de um crescente número de casos nos últimos anos, com um aumento dos índices de diagnósticos de 1 em cada 2.500 pessoas em 1978, para 1:100 pessoas em 2014 (Hollin, 2014; Eyal et al., 2010). Tais dados, como comentei, têm gerado debates internacionais sobre uma provável “epidemia de autismo” (Hacking, 2006; Grinker, 2010; Eyal et al., 2010; Hollin, 2014; Rios et al., 2015), cujas teses não descartam o fato de que quanto mais se sabe sobre uma doença ou deficiência, mais se tem ferramentas para diagnosticá-la, e mais pessoas identificam-se sob aquela classificação.

Como lembra Ian Hacking (2006), as investigações científicas interagem com as classificações e as mudam constantemente, processo de construção social chamado pelo autor de looping effect das categorias. A cada looping effect, não só uma nova categoria é criada, mas também novas pessoas são nelas incluídas e as pessoas classificadas são recriadas, não sendo mais as mesmas que anteriormente. No caso do autismo, o percurso histórico de aquisição de conhecimento sobre a condição conta com o fato de que a crescente amplitude de "sintomas" e "sinais diagnósticos" incluídos no chamado Transtorno do Espectro Autista (TEA) ${ }^{11}$ dá margem a sua abertura a diferentes interpretações e o torna adaptável a um número crescente de pessoas (Grinker, 2015).

Segundo Hacking (2006:4), "em 1973 o autismo era um distúrbio de desenvolvimento raro, com um estereótipo bastante definido e estritamente caracterizado. Hoje nós temos o Espectro do Autismo. Nós temos pessoas com autismo de alta-funcionalidade. Nós temos Aspergers"12. O autor cita exemplos de pessoas adultas que hoje se identificam como autistas ou Aspies, mas que, devido à configuração do autismo ser mais desconhecida e estreita quando eram crianças, cresceram sem um diagnóstico específico sobre sua condição ${ }^{13}$.

Em uma das entrevistas que realizei, comentei com um psiquiatra que achava que alguns conhecidos meus poderiam ser identificados como autistas dentro dos padrões de comportamento que ele relatava. Nesse momento, ele me disse:

Isso é muito comum. Vários adultos cresceram achando que eram estranhos ou não sabendo o porquê das pessoas não gostarem deles e porque nunca se sentiram iguais às outras pessoas. Daí alguém fala pra eles sobre o autismo e eles descobrem que têm o diagnóstico. Isso acontece muito também com os pais de autistas nas consultas dos filhos, quando começo a falar as características do autismo e eles se identificam. 
Segundo especialistas, além dos diagnósticos infantis terem-se multiplicado, cada vez mais adultos estão se identificando e sendo identificados nesta classificação.

Este aumento de diagnósticos conta também com uma positivação do Espectro, impulsionada em parte por um crescente número de séries e autobiografias (Hacking, 2009), mas também pelo crescimento do Movimento da Neurodiversidade (Ortega; Choudhury, 2011) no mundo. No Brasil, e especialmente no Rio Grande do Sul, esse movimento é pouco conhecido, inclusive entre alguns especialistas da área psi com quem conversei. Mas, na internet, Rios e Andrada (2015), pesquisadoras do Rio de Janeiro, encontraram uma crescente rede atuante neste sentido.

Por outro lado, alguns elementos do processo parecem indicar uma certa dificuldade na obtenção do diagnóstico. Como a psicopedagoga Clara e também alguns neurologistas salientaram em nossas conversas, o fato de o autismo não ter um "exame médico" que o identifique, um "biomarcador", e nem uma "marca corporal” visível faz com que médicos relutem em dar certeza deste diagnóstico, considerado por eles como "muito subjetivo". Além disso, segundo eles, pelo menos aos olhos de desconhecedores do transtorno, não há como identificar - diferentemente da Síndrome de Down, por exemplo - “o que a pessoa tem”. Os sinais são sutis, porque comportamentais, e exigem um conhecimento sobre o que é o autismo e um "olhar treinado" para que sejam perceptíveis. Segundo eles, tal fato somado à incipiente formação acadêmica em autismo dificulta o acesso ao diagnóstico.

A dificuldade de "fechar um diagnóstico de autismo" se daria, então, tanto por conta do pouco conhecimento que ainda se tem sobre a condição (Fitzgerald, 2015; Hollin, 2014), mas também por ser um processo diagnóstico considerado demasiado "subjetivo" pela classe médica (Grinker, 2010), ou, nas palavras dos especialistas, um “diagnóstico clínico".

\section{Autismo: um “diagnóstico clínico”}

Ao mesmo tempo em que há um discurso médico bastante calcado em uma concepção positivista de ciência que acusa a pouca "cientificidade" do processo diagnóstico do autismo, as habilidades pessoais "subjetivas" dos experts na sua identificação são frequentemente exaltadas. Ou seja, a especificidade de realização de um diagnóstico a partir de observações do comportamento das pessoas, da forma como interagem e se comunicam contribui para uma disputa em torno da sua classificação, tornando o autismo o que poderíamos chamar, seguindo Rosenberg (2002), de uma "categoria perigosa". 
Gregory Hollin (2014a) analisa as especificidades da construção social do diagnóstico do autismo nas experiências profissionais de especialistas no assunto e comenta que vários de seus interlocutores lhe disseram - assim como eu também ouvi algumas vezes em meu campo - que "são capazes de determinar se um indivíduo é autista segundos após a sua chegada na clínica" (Hollin, 2014a:99). "Eu boto o olho e já sei que é autista”, escutei de mais de um especialista. Tais comentários, no entanto, ponderavam a "intuição" com argumentos de "seriedade" e "cientificidade" da profissão, como: "Claro que depois sigo os protocolos, faço vários testes validados cientificamente”; ou: "mas é necessário muitas consultas e várias avaliações para fechar um diagnóstico sério”.

Tais "testes validados cientificamente" utilizados na produção de diagnósticos têm sido objeto de análise e críticas por parte da Sociologia e da Antropologia Médica e Psicológica ${ }^{14}$ já há algumas décadas. Em uma análise recente sobre a produção diagnóstica específica do autismo, a partir do teste ADOS (Autism Diagnosctic Observation Schedule), Elizabeth Fein (2018) argumenta, por exemplo, que, na verdade, esse teste parte de uma avaliação da habilidade das pessoas de performarem o que Giddens (1993) chama de pure relationship. Segundo Fein (2018: 145), a existência de um "self puro individual" e de agir dentro de normas sociais precisas, mas demonstrando estar agindo "espontaneamente" e "livres de pressões socioculturais", além de serem "imperativos culturais" incompatíveis com a forma autista de engajamento social, são uma forma arbitrária e descontextualizada de avaliar a condição. Jurandir Freire Costa e Roy Richard Grinker (2018) também contribuem para o debate com uma análise à luz da filosofia da linguagem e da fenomenologia, sobre como os autistas constroem suas personalidades e vocabulários interpretativos de forma coerente, chamando a atenção para o fato de que "a cognição não é um processo desincorporado a ser julgado nos termos de um consenso neurotípico" (Costa; Grinker, 2018: 160), evidenciando como os "discursos de autistas são também formas de resistência a discursos hegemônicos de autoridade” (op cit:171).

Nessa disputa de saberes, a reivindicação das mães de autistas ${ }^{15}$ (Rios, 2018; Nunes, 2014; Ferreira, 2015) na validação de seus conhecimentos sobre os filhos é uma das especificidades sobre esta condição. Em meu campo sobre a inclusão de pessoas com deficiência pela política de cotas, algumas foram as vezes em que escutei dessas mães que elas "sabiam que seus filhos tinham autismo", mas que "os médicos não davam o atestado" ${ }^{16}$ para que eles tivessem acesso à vaga de emprego pelas cotas. Na maioria das vezes, essas pessoas tinham "traços leves" que os situa- 
vam no Espectro e não havia outras "comorbidades" (doenças ou deficiências além da preponderante) com um CID de uma deficiência legalmente válida.

Certo dia, na saída do curso de capacitação onde Clara trabalhava, presenciei uma mãe de um jovem com autismo que se lamentava para uma professora que “a empresa não aceitou o CID de seu filho". Segundo ela, "lá dizia só que ele tinha TDAH" 17 , mas que ela "sabia que o filho tinha mais que isso". Segundo a professora, esse atestado realmente não se enquadrava nas regras da política de cotas, mas que ela concordava com a mãe sobre o filho "parecer ter algum grau de autismo" e que "conhecia alguns médicos mais familiarizados com o espectro para tentar uma consulta para o rapaz”.

As disputas em torno do diagnóstico de autismo também estavam presentes, mas de outra forma, no processo de Tomás, um dos jovens com quem convivi durante minha pesquisa e cujo processo de inclusão Clara assessorou. Clara contou-me que Tomás havia chegado no curso com o "F7.5, diagnóstico de retardo mental leve", mas que "já de cara" a Renata, sua colega de trabalho, que "tá com um olho bom", havia lhe dito: "Esse menino é estranho". Daí, quando ela foi avaliar, percebeu que era "óbvio que ele era autista". Ela falou com Joana, a mãe do rapaz, e fez uma avaliação. Segundo Clara, ao verificar outros documentos trazidos pela mãe, verificou que Tomás já tinha o diagnóstico F84.0 - “Autismo Infantil” - feito aos 7 ou 8 anos, quando estava na escola.

Mas para Joana, mãe do rapaz, assim como para Tomás, que se refere a si mesmo como "pessoa que tem dificuldade" - tal papel com letras e números não dizia muito sobre seu filho. Joana percebia Tomás em contraste com o filho mais novo, que tinha ecolalia $^{18}$ e limitações para realizar atividades fisiológicas diárias, cujo diagnóstico, para ela, era sim de autismo. Joana me explicou que Tomás não era como o irmão, tinha "apenas uns por cento de retardo lá". Aquele atestado do médico representava para ela apenas um documento que o filho precisava para ter acesso a uma vaga de emprego.

Trago estas controvérsias não para levantar a questão de quem sabe mais sobre Tomás ou qual diagnóstico está certo ou errado. Dizer que Tomás não habita a categoria autismo ou que sua mãe não o reconhece nesse diagnóstico não deslegitima o saber médico, mas nos fala sobre a especificidade desta categoria e sobre como as pessoas experienciam, agenciam e convivem com os rótulos biomédicos. Parece que ao escolher "retardo" ao invés de "autismo", a mãe de Tomás está indicando mais um elemento que compõe o "perigo" da "subjetividade autista": sua compreensão de 
que o autismo representaria uma "deficiência mais incapacitante" do que a que ela via na condição de seu filho, ou, pelo menos, com menor probabilidade de contratação pelo mercado de trabalho.

Como bem lembra Rosenberg (2002), diagnósticos são meios de comunicação, campos de negociação que configuram e reconfiguram vidas e criam alianças. Evidentemente que toda disputa é situada na História e não se dá em termos de igualdade de condições. No caso do diagnóstico do autismo, cabe ainda pontuar que, nas disputas em torno de sua classificação, o papel dos saberes biomédicos (em detrimento dos psicanalíticos) ocupa um espaço central. Na prática da política, a presença de médicos neurologistas e psiquiatras é marcada não apenas pelo poder da produção da verdade na construção do laudo diagnóstico que dá acesso às cotas, mas também na prática cotidiana das pessoas envolvidas na implementação da política.

\section{Os "experts da subjetividade" em disputa}

O poder das categorias biomédicas como detentoras de um saber legítimo sobre a saúde e a doença, sobre corpos e mentes, é incontestável em nossa sociedade. Especificamente em meu campo de pesquisa, para fins da Lei de Cotas, laudos diagnósticos são emitidos apenas por médicos neurologistas ou psiquiatras (Nubila, 2007). Nesse documento deve constar uma "deficiência" diagnosticada com um número da CID. Tal caracterização encontra-se no Decreto 5.296/04, cujas concepções de deficiência são preponderantemente biomédicas (Nubila, 2007).

Segundo Nikolas Rose (1997; 2008; 2013) e Ortega (2008; 2010), nossas características subjetivas estariam cada vez mais sendo interpretadas como localizadas no cérebro físico, sendo, então, a nossa própria subjetividade entendida como neuroquímica. Evidentemente que essa mudança de "estilo de pensamento" (Rose, 2008) não é vista como um processo evolutivo. Uma forma de pensar não substitui a outra, mas se soma às outras já existentes e torna-se hegemônica em certo tempo histórico. Saberes estão sempre em disputa e o que vemos nos debates em torno do autismo hoje, apesar das disputas com a psicanálise no Brasil serem presentes (Lopez \& Sarti, 2013; Ortega et al., 2013), é uma preponderância dos saberes biomédicos na explicação, e principalmente no "manejo" e no "tratamento", desta condição ${ }^{19}$ (Hacking, 2006; Ortega, 2008; Campoy, 2015).

Segundo Ian Hacking (2006), o autismo, que já foi visto como o comportamento de pacientes esquizofrênicos ou "uma desordem psiquiátrica da infância", hoje é objeto de disputas entre os que o entendem como uma desordem do desen- 
volvimento, uma deficiência ou uma diversidade neurobiológica (Hacking, 2006; Ortega, 2008). Em termos gerais, tais nomenclaturas (desordem, transtorno, deficiência, neurodiversidade) carregam diferentes concepções sobre as possíveis causas do autismo, suas características e manifestações físicas, intelectuais, sensoriais e atitudinais, assim como informam as posturas dos experts frente à melhor abordagem e aos possíveis tratamentos/curas (Solomon, 2013; López \& Sarti, 2013; Campoy, 2015) para esta condição.

A passagem do autismo da lista de "psicoses infantis" para sua incorporação aos "transtornos do desenvolvimento", mencionada por Ian Hacking, se deu na terceira edição do DSM. Esta mudança refletiria a chamada "virada fisicalista”, que exporia, também num nível mais pragmático, as mudanças nos sistemas de pensamento mencionadas por Rose, com o deslocamento das concepções psicanalíticas sobre o autismo para o predomínio de uma concepção biológica e cerebral do transtorno.

A ideia de cunho psicanalítico de que o autismo seria causado por uma "falha dos pais no estabelecimento de relações objetais precoces com o filho" (Ortega, 2010:150) foi, então, superada pela concepção hegemônica de que ele estaria localizado no cérebro. Com isso, as metáforas da "mãe geladeira", "conchas", "tomadas desligadas", que tanto culpabilizavam as mães, foram rechaçadas pela maioria dos especialistas (Eyal \& Hart, 2010; Nunes, 2014) ${ }^{20}$. Não é à toa que todos os especialistas que conheci que trabalhavam com autismo, como Clara e Pedro, ou eram neurologistas e médicos psiquiatras, ou eram da linha behaviorista ou cognitivo-comportamental da psicologia, em geral, críticas à psicanálise freudiana (López \& Sarti, 2013).

Há hoje, como mencionei no início deste texto, um consenso entre os especialistas de que, na prática, o autismo se traduziria em "dificuldades de comunicação e socialização”. Estas dificuldades seriam a não compreensão da obrigatoriedade arbitrária e subjetiva de seguirem regras sociais implícitas em situações cotidianas (muitos dizem que eles não têm "filtro social") e de compreensão de sinais culturais e sociais da comunicação, principalmente a comunicação não verbal (como entrelinhas, metáforas e expressões faciais). Tais “características”, apesar de marcarem uma imprevisibilidade de seus comportamentos em sociedade - mais uma marca de sua "subjetividade perigosa" -, não asseguram um consenso entre os especialistas a respeito de suas subjetividades ou sobre "quem são" essas pessoas. 


\section{Diversidade cerebral ou deficiência?}

No Brasil, Rios e Andrada (2015) apontam que o processo de construção diagnóstica do autismo é constituído por um duplo campo de tensões: teóricas e políticas. Seu status nosológico mais do que ser objeto de disputas teóricas - em torno de sua definição como uma condição orgânica ou psicológica - é palco de disputas políticas quanto à sua definição como uma "deficiência” ou uma "diferença”. Eu diria que estas duas questões se intersectam, e que os julgamentos morais que estas concepções carregam estão informados tanto pela possível causa do autismo quanto pela sua classificação.

Neste debate, os interesses acadêmicos voltam-se para a análise do surgimento de grupos de ativistas que entram no campo de disputas pela expertise na área, com a participação, por um lado, de autistas que reivindicam sua identidade de "neurodiversos" (Ortega, 2008; 2010; Fein, 2011) e, por outro, como já mencionei, de grupos de "mães especialistas" (Nunes, 2014; Eyal \& Hart, 2010; Rios, 2018; Hart, 2014), com crescente presença nas redes sociais (Ortega et al., 2013), que denunciam a condição "discapacitante" do autismo e reivindicam o acesso aos benefícios e políticas sociais para seus filhos.

Na visão desses atores, principais envolvidos nesta questão, o reconhecimento do autismo para todos os fins legais como uma "deficiência" ${ }^{21}$, por um lado, proporcionaria às pessoas diagnosticadas no espectro o acesso aos benefícios e políticas sociais, mas, por outro, acarretaria na incorporação de todos os estigmas de "déficit", "incapacidade" e "não autonomia" que esta categoria carrega em nosso senso comum. Por este motivo, tal nomenclatura é rechaçada por aqueles que advogam ser o autismo uma "neurodiversidade" (Ortega, 2010; Ortega \& Choudhurry, 2011; Fein, 2011), desobrigando as pessoas a vestirem o rótulo da "deficiência".

Apesar de minha pesquisa mostrar as barreiras simbólicas que essas pessoas enfrentam em seu dia a dia, esta "fatia" específica de pessoas com autismo que teria condições de levarem suas vidas sem grandes limitações é apenas uma parte pequena da camada da população situada no Espectro. Daí as reivindicações das cuidadoras para que o autismo seja considerado uma "deficiência".

Tais disputas indicam como os rótulos são complicados e o fato de "habitá-los" (ou não) têm efeitos práticos e simbólicos na vida e na construção de subjetividades das pessoas. Como comentei anteriormente, apesar de Tomás e sua mãe não nomearem a sua condição de "autismo", as categorias diagnósticas aparecem como centrais para compreensão da construção da sua subjetividade. Ao final do período 
de estágio na empresa, ele comentou comigo que "agora era um trabalhador" e que seu chefe havia lhe dito que "agora nem parecia mais que tinha dificuldades". Tal transformação subjetiva só pôde se efetivar, pragmaticamente, por conta de ter um diagnóstico válido para ter acesso às políticas sociais e, simbolicamente, por este “diagnóstico" (seja qual for) ter perdido a sua centralidade frente à nova realidade vivida no mundo do trabalho. Sua experiência mostra que, mais do que saber ou "aceitar" se tem autismo ou não, ou “incorporar" esta nomenclatura à sua identidade ou não, esta categoria é acionada ou ignorada ao longo de sua trajetória em situações diferentes e por diferentes atores e motivações. Suas ações e relações estabelecidas com os diversos atores que participaram de seu processo de inclusão transformaram o que é o "autismo" e "como é uma pessoa com autismo" ao longo de seu percurso, evidenciando o looping effect (Hacking, 2006; 2007) dessa categoria.

Autores como Rosenberg (2002) e Hacking (2006) têm mostrado que o processo de construção diagnóstica é histórico e complexo, e envolve não apenas saberes e poderes médicos, mas o papel ativo das pessoas diagnosticadas na construção destas categorias e das subjetividades envolvidas em sua nomeação. Ian Hacking (2006) analisa o processo de classificação das pessoas (o qual ele chama de making up people), mostrando como e por quem as pessoas são classificadas, como as classificações as afetam e, também, como estes efeitos das classificações nas pessoas modificam as próprias categorias (o que ele nomeia de looping effect das categorias). Tanto Rosenberg quando Hacking entendem que o ato de "diagnosticar" vincula a pessoa ao sistema social e tem, ao mesmo tempo, os efeitos de "rotular" e de "libertar”. Nas palavras de Rosenberg (2002:254-257), no momento da identificação diagnóstica, "uma cortina é retirada, e a incerteza é substituída - para melhor ou para pior - por uma estrutura narrativa”, na qual "ansiedade e mistério podem ser ordenados no sistema social”, fazendo do diagnóstico "uma necessidade emocional e burocrática” que atua na subjetificação das pessoas.

\section{Uma subjetividade autista?}

Em meu campo, a preocupação com a dessubjetificação das pessoas frente ao rótulo do diagnóstico não era apenas minha. Esta questão - reforçada pela especificidade do diagnóstico de autismo levar consigo a não presença de uma subjetividade empática - estava em jogo ao longo de todo meu trabalho de campo e nos diversos espaços por onde circulei.

Uma das queixas mais frequentes que escutei de agentes das políticas públicas 
com relação à inclusão de pessoas com deficiência nas empresas é a de que "elas não contratam pessoas, elas contratam deficiências”. Ao invés de fazerem um processo seletivo com foco nas competências e nas habilidades de cada candidato, elas já abrem as vagas direcionadas para uma deficiência específica ${ }^{22}$. Pessoas cegas são telefonistas, surdas trabalham em espaços de alto impacto de ruídos (como fábricas e pistas de aeroportos) e pessoas com Síndrome de Down são empacotadores de supermercados, comentou em entrevista ao rádio um fiscal da política de cotas. "Eles esquecem que ali tem uma pessoa", salientou Clara na saída de uma de suas visitas de consultoria, "me irrita ver como sempre julgam as capacidades deles pelo diagnóstico".

Ver a pessoa em primeiro lugar e não generalizar e homogeneizar as pessoas com deficiência são reivindicações que perpassam os debates dos Disability Studies e a história do Movimento Político das Pessoas com Deficiência. Desde a sua nomeação, passando pelas concepções do que é deficiência, até as suas lutas por direitos, tais atitudes são combatidas por terem um caráter capacitista ${ }^{23}$ (Campbell, 2009; Mello, 2016). No entanto, apesar dos esforços em ter a pessoa como foco (ou em primeiro plano), parece que o poder do sistema biomédico de pensamento e o encantamento pelo "funcionamento do cérebro", que perpassara toda a formação e a vida profissional dos médicos, são hegemônicas em nossa sociedade. Um fascínio pelas neurociências como base de um sistema de pensamento de explicação de si (Rose, 2008) é evidente entre esses profissionais. Quando falam, então, que "quando tu conheces uma pessoa com autismo, tu conheceu uma pessoa com autismo!"24, indicam não apenas que devemos prestar atenção na pessoa como um indivíduo único, mas que não há como generalizar as características do diagnóstico neurológico em si. E, nesta multiplicidade de sujeitos dentro de uma única nomeação diagnóstica, vemos mais uma especificidade desta "categoria perigosa".

Foram muitas as vezes que senti como se os médicos neurologistas e psiquiatras, e também Clara, estivessem falando do cérebro como uma entidade autônoma e com vida própria. Como no dia em que ouvi este comentário de Pedro, um psiquiatra especializado em autismo, em uma de nossas conversas:

Trabalhar com pessoas com TEA é como sintonizar uma rádio naqueles aparelhos antigos. Tem que girar o botão devagar, com precisão, até pegar bem a frequência (...) às vezes leva meses para que a gente consiga entrar em sintonia e começar uma troca, às vezes pode ser instantâneo. 
Pedro já acompanhou a inclusão de vários pacientes no mercado de trabalho e comenta que "o problema é que as empresas não estão prontas para lidar com pessoas com autismo”. A grande maioria, diz ele,

tem dificuldade de aceitar e até de entender que o autista não vai mudar, ele não vai desenvolver muitos dos comportamentos exigidos no mercado de trabalho (...) Depois de um tempo trabalhando na empresa, tu não vais pedir para um cadeirante levantar, mas as pessoas esperam que o autista possa começar a se entrosar, ou possa mudar de departamento e atender o público, por exemplo.

Essa fala de Pedro expressa a necessidade de adaptação da sociedade para incluir as pessoas com deficiência, mas também reafirma a concepção do autismo como uma condição cerebral, situada no campo do biológico e, portanto, "sem cura", sem possibilidades de mudança. Tal ideia, paradoxalmente (ou não!), é compartilhada pelo Movimento da Neurodiversidade, (Ortega, 2008; 2010), o qual considera o autismo como uma outra forma de ver, viver e estar no mundo.

$\mathrm{Na}$ interpretação dos especialistas e, também, dos Aspies, com quem convivi ou conversei, a própria subjetividade das pessoas com autismo parecia ser entendida, até certo ponto, como constituída biológica ou neurologicamente. "Não somos doentes, não temos déficits ou deficiências, somos neurodiversos". "É a sociedade que não tem a capacidade de nos entender e de se comunicar conosco", dizem os ativistas deste movimento.

Inicialmente, num olhar das ciências humanas, como bem indicam Dias Duarte (2018) e Ortega (2008), esta "biologização dos sujeitos" por parte dos ativistas neurodiversos levaria a uma crítica ao reducionismo presente na compreensão do humano como totalmente "fisicalista" ou "naturalista", típica de uma visão biomédica que acarretaria numa concepção reducionista e desubjetivante de pessoa e numa concepção de subjetividade que não concebe as categorias como relacionais (Campoy, 2015). No entanto, no caso do autismo, essas críticas devem ser problematizadas, pois, neste caso, há uma certa positivação da condição e uma compreensão desta "biologia" na chave interpretativa de uma "diversidade humana", que é, de certa forma, não hierárquica e não patologizante. Por outro lado, se é verdade que há um esvaziamento do sujeito na biologização da compreensão da "subjetividade autista”, há também uma ressubjetivação específica destas pessoas a partir dos próprios "traços" do autismo, como constituidores de um "sujeito moral". 
Luiz Fernando Dias Duarte (1983) desenvolve com densidade a constituição do sujeito moderno como a unidade significativa do valor atribuído ao indivíduo: "sujeito da razão, da história e da moral" (1983: 7). Desenvolver um debate aprofundado sobre as moralidades que envolvem a produção de uma "subjetividade autista" vai além do espaço deste texto, no entanto, cabe indicar que a atribuição de características morais ao "cérebro diverso" poderia indicar uma exacerbação da individuação, própria do sujeito moderno analisado por Duarte. Para os objetivos centrais deste texto, cabe perceber que esta ideia fisicalista sobre quem é o sujeito com autismo, além de não ser consenso nas disputas entre experts, tem efeitos práticos na inclusão destas pessoas no espaço laboral, no sentido de que, apesar de positivada, esta subjetividade pode tornar-se "exótica".

Um dos neurologistas do Hospital de Clínicas de Porto Alegre, em entrevista ao programa de TV Vida e Saúde (2013), afirma que: “os autistas são ingênuos, bons e sinceros. Eles são incapazes de dizer uma mentira. Não porque querem, mas porque eles não podem ser de outra forma", e finaliza sua fala afirmando: "Se você quer uma pessoa honesta, contrate um autista". Em uma de nossas conversas, também o psiquiatra Pedro tenta me explicar esta "especificidade" dos autistas, dizendo que "é como se eles tivessem uma forma infantil de pensar. Não têm filtro social, sabe? Dizem o que querem, quando querem. Parecem crianças na fase dos porquês, já que são desconhecedores da maldade e das regras sociais, então cometem gafes seguidamente" (Pedro, entrevista em abril de 2015).

Na prática cotidiana das empresas, as "gafes" a que se refere Pedro, muitas vezes narradas como engraçadas, nem sempre (ou quase nunca) são compreendidas como sendo "coisas do autismo" e, mesmo quando são, não são bem recebidas. Foram várias as histórias anedóticas que presenciei e escutei ao longo da minha etnografia que ilustram "desentendimentos" entre neurodiversos (autistas) e neurotípicos (não autistas). Foram muitos também os momentos de tensão e as injustiças e violências que presenciei devido à incompreensão desta outra forma de estar no mundo.

\section{O cotidiano nas empresas}

Ao longo de minha etnografia com Tomás, convivi com ele por seis meses na empresa na qual teve sua primeira experiência de trabalho. Durante esse tempo, sua característica de "não solicitar nova tarefa ao ter acabado as atribuições que lhe tinham delegado" e "não se entrosar com as pessoas" foram seguidamente apontadas como possíveis causas de sua não contratação após o período de experiência. 
"Hoje em dia temos que vestir a camiseta, construir boas relações com os colegas. Se ele continuar assim isolado não sei se fica”, comentou um colega de trabalho. "Ele fica ali paradão, não tem iniciativa de procurar o que fazer", além de "não falar com ninguém, almoçar sempre sozinho”, me relatou outra funcionária do setor em que Tomás trabalhava, seguindo com a pergunta: “Mas afinal, o que ele tem, hein?".

Além desses traços, Tomás, aos olhos dos colegas, parecia frágil e representava, de certa forma, um "perigo" no ambiente de trabalho: alguém cujas atitudes eram imprevisíveis e com as quais os colegas poderiam não saber lidar. Seu Luís contou-me que "um dia congelou em frente às prateleiras do depósito, ficando lá imóvel por alguns minutos", chamando a minha atenção para o perigo que era ter um rapaz como ele naquele espaço. "E se vem uma empilhadeira? Eles andam rápido naquelas coisas! E se ele, de uma hora pra outra, tem um troço lá? Perigoso isso”, alertou.

No caso de Tomás, a empresa não havia feito o que no espaço empresarial se chama "sensibilização dos funcionários" para a chegada de uma pessoa com deficiência. Seus gestores e colegas não sabiam do diagnóstico de Tomás, o que fez com que nos primeiros meses, as fofocas sobre "seu autismo" fossem assunto pelos corredores da empresa. Aos poucos os colegas foram "se acostumando" com o jeito do menino e alguns pesquisando na internet os "sintomas" da sua condição, explicavam o porquê de Tomás ser daquele jeito para os demais.

Tomás não falava muito, então, as "gafes" mencionadas por Pedro eram menos frequentes, mas num mercado que exige "pró-atividade" e "inteligência emocional”, tais "qualidades do autismo" colocam barreiras muitas vezes intransponíveis se a contratação das pessoas com este diagnóstico não pressupõe uma concepção de processo inclusivo.

Tais "gafes", geralmente identificadas como "próprias dos autistas", também eram corriqueiras no cotidiano de outro interlocutor de minha pesquisa. Durante os quatro meses em que estive na empresa onde Ricardo trabalhava, escutei várias histórias sobre as "suas gafes". A gerente do setor onde ele trabalhava contou que

o Ricardo é um sarro. Um dia a gente pediu que ele distribuísse os convites da reunião com a diretoria para os colaboradores. Ele perguntou quais funcionários. Daí eu disse para ele distribuir para todos os setores. Ele deu convite pros porteiros, faxineiras pro pessoal da segurança... E todos foram na reunião geral! Sabe que isso fez com que caísse uma ficha na gente, né! Ele nos deu uma aula de horizontalização da gestão ali! (Gerente do Ricardo, agosto de 2014). 
Estes mal-entendidos, no entanto, não geravam apenas situações jocosas ou resultados "positivos". Momentos de tensão também faziam parte do cotidiano das relações de Ricardo com os colegas na empresa. Frequentemente sobrecarregados de atividades extras, eles tentavam, sem sucesso, dividi-las com Ricardo. Ao ser solicitado, ele seguia à risca suas atribuições, dizendo que "aquela tarefa não estava prevista em seu cronograma semanal", ou "Ah, não, essas coisas são muito chatas de fazer".

Tais situações foram entendidas por alguns colegas como "coisas do autismo" e por outros como "resultado do mimo excessivo da mãe de Ricardo" ou "esperteza do guri". Clara disse que é bem comum as pessoas se irritarem ou não entenderem esse jeito de eles se comportarem. "Se tu falas que as tarefas são tais, ele vai seguir aquele protocolo". Em um dia em que fizemos várias visitas às empresas que estavam com "casos de inclusão de autistas", presenciei mais algumas destas situações de tensão, nas quais julgamentos morais estavam presentes:

Um dia, um menino que trabalhava em um supermercado foi "flagrado" com vários pacotes de bolacha na sua mochila, quando a verificaram na saída do turno. $\mathrm{O}$ gerente o acusou de roubo e foi um bafafá... O menino disse que havia guardado as bolachas para os colegas porque nas bolsas deles não tinha espaço. Daí me chamaram pra resolver o problema. Eu disse pro gerente que ele acreditasse no menino. Eles não mentem. Se ele disse que foi assim, é porque foi isso que aconteceu. Eu conheço eles [as pessoas com autismo]. (Clara, agosto de 2014).

Uma outra vez foi parecido. Alguém no super reclamou que tinha esquecido o celular em casa e que ficava perdido sem saber as horas e dar uma olhadinha na internet de vez em quando. Não é que o guri diz o seguinte: "Ah eu te dou um. Tem um monte no meu armarinho!”. Um colega roubava os celulares e pedia pro guri guardar pra ele. (Clara, agosto de 2014).

A Maria tem dado bastante problema lá no hospital. Volta e meia me chamam pra conversar com ela e com as colegas. Ela senta no chão dos corredores quando tá cansada; entra na sala da diretora pra conversar e fica lá de papo, come os iogurtes das refeições dos pacientes... E também as colegas de trabalho não conseguem lidar muito com ela. Quem gosta de escutar, assim na lata, as verdades da vida, né? Porque ela chega e diz: "Olha, tu tá mais gorda. Dá pra ver a barriga” ou "Eu não gosto de ti. Tu é bem chata”. (Clara, setembro de 2014).

Tais situações, apesar de gerarem mal-estar e tensão no cotidiano das empre- 
sas, não tiveram punições severas como resultado. Já outros casos que escutei em conversas com os jovens com autismo que conheci, isso não ocorreu. Fabian, um jovem de 24 anos, diagnosticado com Asperger, por exemplo, contou-me que em um de seus primeiros empregos, elogiou a beleza da sua chefe dizendo que "gostaria de namorar com ela”. Segundo ele, tal situação, somada a outras similares, nas quais "estava apenas falando a verdade de como se sentia”, lhe renderam um processo de danos morais. "Não adiantou eu dizer que era autista. Eles não levaram minha condição em consideração". Fabian me conta que ele nunca diz que é autista antes de entrar em um emprego. Se o fizesse, não seria contratado. Mas, por outro lado, reconhece que se não expõe sua condição, não tem acesso a um tratamento diferenciado.

\section{Sobre deficiência, autismo e políticas de inclusão}

Ao longo de toda a minha pesquisa de campo no Rio Grande do Sul, meus interlocutores pouco mencionaram uma possível “epidemia de autismo”, mas os discursos das mães de autistas na internet e de especialistas nos eventos nacionais e internacionais que participei traziam números alarmantes e salientavam a importância da comunidade médica e acadêmica direcionarem seus olhares para esta condição. $\mathrm{O}$ autismo se tornava, em seus discursos, uma urgência médica a ser conhecida e atendida pelo poder público, ao mesmo tempo em que se transformava em uma nova fatia de mercado ainda não explorada para os profissionais da medicina e psicologia. O discurso da "epidemia” legitimava o autismo como uma questão pública tanto na busca por recursos de pesquisa quanto na obtenção de direitos sociais.

Neste texto, procurei mostrar alguns elementos que estão em jogo nesta questão. O caráter fluido e subjetivo da construção do diagnóstico de autismo e a maior amplitude que a mudança do seu status nosológico para "espectro do autismo", assim como a crescente midiatização do fenômeno, podem ter dado margem a um crescente número de pessoas que se enquadram no espectro, contribuindo para a legibilidade quantitativa da condição, fundamental na conquista de espaço na agenda política.

Trouxe também para o debate o fato de que os efeitos desta legibilidade podem estar na aprovação da lei Berenice Pianna e de novas políticas públicas específicas para esta população, como, aqui no Rio Grande do Sul, na criação do projeto Autismo e Mercado de Trabalho. Por outro lado, também a Lei, ao dar maior visibilidade ao autismo, teve efeitos na crescente onda de diagnósticos, agora legitimados 
frente ao acesso aos direitos sociais dela advindos. Não há "números" para auxiliar na reflexão sobre este assunto. O que posso afirmar é que, se essa questão aponta para possíveis efeitos em um nível "macrossociológico" na minha pesquisa, e aparece apenas superficialmente em meu campo, em um nível mais micro, são as formas de compreensão sobre o que é o autismo em si e sobre como lidar com essas pessoas no cotidiano dos processos de inclusão que ganham maior espaço.

Como mencionei, uma das críticas aos processos de recrutamento e seleção das empresas é o fato de elas "contratarem a deficiência e não a pessoa”. No mercado de trabalho, alguns diagnósticos são mais "bem-vindos" que outros na valoração das vidas (Fassin, 2009) - e nas empresas parece ficar o caráter produtor de "subjetividades perigosas" do autismo. O perigo da incerteza do diagnóstico; o perigo de não ter como tipificar "um perfil comportamental"; o perigo de não poder prever como autistas reagirão em situações de tensão ou não rotineiras colocam barreiras atitudinais bastante distintas das encontradas na inclusão de pessoas com deficiência que necessitam de mudanças nos espaços físicos e/ou de tecnologias assistivas, e, por isso, mais difíceis de serem derrubadas.

Além disso, o pouco conhecimento sobre o espectro do autismo, o caráter "clínico" do seu processo diagnóstico e as disputas em torno desta categoria por parte de especialistas também dificultam a obtenção de um laudo que dá acesso às políticas públicas. E o desconhecimento das chamadas características específicas dos neurodiversos pela população em geral levam a julgamentos morais de responsabilização da mãe e dos próprios autistas por suas atitudes "pouco simpáticas" ou "ingênuas", levando muitas vezes a confrontos e momentos de tensão em seus cotidianos.

Com isso, quero salientar que é nas interações sociais que as diferenças de comunicação e interação entre neurodiversos e neurotípicos aparecem e mostram seus efeitos. Romper a barreira que impede o acesso das pessoas com autismo à participação plena na vida social implicaria, então, no questionamento das normatividades impostas na nossa forma de nos comunicarmos e interagirmos com as pessoas, entendendo-as como mais uma entre tantas outras maneiras de estar no mundo.

Nesta tarefa reflexiva, etnografias mostram-se fundamentais para uma compreensão aprofundada tanto do autismo em si, como mais uma entre tantas diversidades humanas, quanto sobre as relações sociais que envolvem pessoas com esta condição. É quando lançamos um olhar mais atento ao cotidiano e à prática das políticas de inclusão que percebemos o quanto direcionar o foco de nossas pesquisas para as pessoas é fundamental. Se em "casos de sucesso" como os de Tomás e Ricardo já 
encontramos tantas barreiras a serem ainda rompidas, novos estudos sobre os casos que "não deram certo" se fazem urgentes para a análise das políticas direcionadas a esta camada da população.

Recebido: 14/02/2019

Aprovado: 05/04/2019

Valéria Aydos é bacharel em Ciências Sociais, mestre e doutora em Antropologia Social pela Universidade Federal do Rio Grande do Sul (UFRGS), com estágio no King's College London, supervisionado pelo prof. Dr. Nikolas Rose. Seus interesses e áreas de pesquisa são os estudos antropológicos sobre Cidadania, Políticas Públicas, Deficiência e Trabalho, buscando perceber as relações entre os modos de governo, a cultura e as subjetividades que se constituem nas dinâmicas dos processos de produção diagnóstica e na inclusão social das pessoas com autismo no mercado de trabalho. Atualmente é pós-doutoranda no PPGAS-UFRGS, aonde atua como professora e pesquisadora vinculada ao Núcleo de Antropologia da Cidadania (NACI), além de participar de Comitês de Assessoramento em Deficiência, Acessibilidade e Inclusão (ANPOCS, ABA, PPGAS-UFRGS). ORCID: 0000-0002-3298-5727. Contato: valeria.aydos@gmail.com

\section{Notas}

1. A pesquisa de doutorado que deu origem a este texto foi financiada pelo CNPq e, posteriormente, contou com bolsa Capes PNPD, junto ao PPGAS-UFRGS. Agradeço a minha orientadora Dra. Patrice Schuch, à colega Helena Fietz, aos colegas do Núcleo de Antropologia do Corpo e da Saúde da UFRGS, especialmente à professora Dra. Ceres Victora, e aos participantes do GT Etnografias da Deficiência (RBA 2018), pela atenta leitura e ricas sugestões.

2. A deficiência é vista aqui de forma relacional (corpos com lesões ou impedimentos em interação com a sociedade na qual existem) e como uma diversidade humana (Diniz, 2012; Ginsburg; Rapp, 2013; Mello; Nuernmberg, 2012).

3. Segundo Ortega (2018), o consenso seria sobre o autismo ser uma neurobiological disorder. Este conceito não implica, porém, em uma ideia comum sobre suas causas e muito menos sobre seu possível tratamento e/ou cura. 
4. A Nosologia é a área da medicina que se dedica ao estudo, descrição e classificação das diferentes doenças.

5. Refiro-me ao Projeto Piloto de Incentivo à Inclusão de Pessoas com Deficiência no Rio Grande do Sul, de iniciativa da Superintendência Regional do Trabalho, e vinculado ao projeto do governo federal Viver Sem Limites.

6. A Lei 8213/1991 estabelece que empresas com mais de 100 funcionários, devem ter de 2 a 5\% de pessoas com deficiência em seu quadro laboral. Ao longo dos processos de inclusão a partir do Projeto de Aprendizado, são três as políticas públicas com as quais meus interlocutores "interagem": o Benefício da Progressão Continuada, a Lei do Aprendiz (L.10.097/00) e a Lei de Cotas (L. 8213/91). Uma análise aprofundada das implicações destas políticas nos processos de inclusão e na vida e na produção de subjetividade de meus interlocutores pode ser encontrada em Aydos (2016) e Aydos \& Fietz (2017).

7. Refiro-me a participantes do "Movimento da Neurodiversidade" e de ONGs de promoção do "orgulho autista” na Inglaterra, com os quais conversei durante o doutorado sanduíche (2015-2016) e/ou pelo facebook.

8. Todos os nomes são fictícios.

9. Por coprodução refiro-me ao que Jasanoff (2004) destaca como sendo a influência mútua dos diferentes atores na construção de uma rede, ao modo como estes atores são induzidos uns pelos outros a agirem.

10. A CID-10 (Classificação Estatística Internacional de Doenças) indica as deficiências reconhecidas em lei para obtenção de benefícios e políticas sociais. Sobre os debates em torno destas produções diagnósticas, ver: Diniz, Barbosa \& Santos (2010) e Mattos (2016).

11. Utilizo o termo biomédico TEA quando me refiro ao diagnóstico médico. Ao longo do texto utilizarei "autismo" por ser a nomeação mais corriqueira em meu campo e abranger várias concepções diferentes sobre a “condição". Utilizo também “condição” ao invés de "transtorno" para marcar minha postura despatologizante com relação ao autismo.

12. A partir da atual edição do DSMV (Diagnostic and Statistical Manual of Mental Disorders), a Síndrome de Asperger situa-se no Espectro do Autismo.

13. Nos debates sobre o Festival "E assim vivemos", de 2013, podemos encontrar algumas narrativas de pessoas diagnosticadas tardiamente com autismo. Disponível em: https://vimeo. com/78497001.

14. Refiro-me aqui, por exemplo, aos vários estudos inspirados pela análise do diagnóstico como um dispositivo de subjetivação (Foucault, 2006); de produção de biossocialidades (Rabinow, 2008) e biolegitimidades (Maluf, 2015).

15. As mães que participam de associações e/ou estão mais envolvidas na busca por direitos de seus filhos autodenominam-se "mães azuis", pelo autismo ser predominantemente um diagnóstico encontrado em meninos (a cada cinco, quatro são meninos).

16. Cascio, Andrada e Bezerra, (2018) mostram que também nos CAPIs existe demora na produção do diagnóstico, mas por outro motivo: a resistência da linha psicanalítica em "rotular a criança" e em identificar o autismo como "deficiência". Para as controvérsias internas no campo psi ver também Rios \& Andrada (2015) e Ortega (2018). 
17. Transtorno do Déficit de Atenção e Hiperatividade.

18. Ecolalia é um distúrbio que causa repetição da fala (palavra ou frase).

19. Cabe comentar que Cascio, Andrada \& Bezerra Jr (2018) indicam uma forte influência da psicanálise nos atendimentos nos CAPSs e CAPSIs brasileiros. No entanto, nas escolas e no mercado de trabalho, a linha comportamental e biomédica é hegemônica.

20. Também foi rechaçada a ideia de que autistas não teriam uma "Teoria da Mente”, ou, em resumo, a capacidade de ter empatia (Silverman, 2008; Ochs et al., 2004).

21. A Lei 12.764, de 2012 foi uma luta política principalmente das chamadas "mães de autistas" (Rios \& Andrada, 2015; Nunes, 2014), lideradas por Berenice Pianna, do Rio de Janeiro.

22. No Brasil, a contratação de autistas é ainda incipiente, mas na Inglaterra é comum vermos autistas em cargos de Tecnologia da Informação. Tal experiência foi um dos destaques de uma feira anual sobre Autismo, chamada de The Autism Show (http://autismshow.co.uk/), da qual participei em 2016.

23. Capacitismo, do inglês, ableism, é um termo utilizado para indicar o preconceito e a discriminação contra a pessoa com deficiência; em geral denunciando o julgamento das capacidades da pessoa a priori e a partir de sua lesão ou impedimento.

24. Esta frase foi repetida diversas vezes em palestras sobre autismo de que participei, e é um dos lemas da organização não governamental Autistic UK, cujas atividades nas redes sociais tenho acompanhado no momento.

\section{Referências bibliográficas}

AYDOS, Valeria; FIETZ, Helena. 2017. When citizenship demands Care. Disability Studies Quarterly, v. 37, n. 4. Disponível em: http://dsq-sds.org/article/view/6087. Acesso em: 30 de janeiro de 2019.

AYDOS, Valéria. 2016. Agência e subjetivação na gestão de pessoas com deficiência: a inclusão no mercado de trabalho de um jovem diagnosticado com autismo. Horizontes Antropológicos. Porto Alegre, ano 22, n. 46, jul/dez, p. 329-358. Disponível em: http://www.scielo.br/pdf/ha/v22n46/ 0104-7183-ha-22-46-0329.pdf. Acesso em: 30 de janeiro de 2019.

CAMPBELL, Fiona. 2009. Contours of ableism: The production of disability and abledness. New South Wales: Springer.

CAMPOY, Leonardo. 2015. Autismo em ação: reflexões etnográficas sem aprovação de comitês de ética sobre a clínica e o cuidado de crianças autistas. Revista de Ciências Sociais Política e Trabalho, n. 42, jan-jul, p. 155-174. Disponível em: http://www.periodicos.ufpb.br/ojs/index.php/politicaetrabalho/ article/view/22803. Acesso em: 23 setembro de 2018.

CASCIO, M. Ariel; ANDRADA, Barbara Costa; BEZERRA Jr., Benilton. 2018. Psychiatric Reform and Autism Services in Italy and Brazil. In: FEIN, Elizabeth; RIOS, Clarice (eds.). Autism in Translation. An Intercultural Conversation on Autism Spectrum Conditions. Culture, Mind and Society, University of Tulsa, USA. p. 53-88.

COSTA, Jurandir Freire; GRINKER, Roy Richard. 2018. Autism and First-Person Accounts: The cognitive problem. In: FEIN, Elizabeth; RIOS, Clarice (eds.). Autism in Translation. An Intercultural Conversation on Autism Spectrum Conditions. Culture, Mind and Society, University of Tulsa, 
USA. p. 155-174.

DIAS DUARTE, Luiz Fernando. 2018. Ciências Humanas e Neurociências: um confronto crítico a partir de um contexto educacional. RBCS, v. 33, n. 97.

DIAS DUARTE, Luiz Fernando. 1983. Três ensaios sobre pessoa e modernidade. Boletim do Museu Nacional. Rio de Janeiro (datilografado).

DINIZ, Débora. 2012. O que é deficiência. São Paulo: Brasiliense.

EYAL, Gil; HART, Brendan. 2010. How parents of autistic children became experts on their own children: Notes towards a sociology of expertise. Annual Conference of the Berkeley Journal of Sociology.

EYAL, Gil et al. 2010. The autism matrix. The social origins of the autism epidemic. Cambridge, Maden: Polity Press.

FASSIN, Didier. 2009. Another Politics of Life is Possible. Theory, Culture and Society, v. 26, n. 5, p. 44-60. Disponível em: https://journals. sagepub.com/doi/abs/10.1177/02632764091063 49. Acesso em: 23 de abril de 2018.

FEIN, Elizabeth. 2018. Autism as a mode of engagement. In: FEIN, Elizabeth; RIOS, Clarice (eds.). Autism in Translation. An Intercultural Conversation on Autism Spectrum Conditions. Culture, Mind and Society, University of Tulsa, USA. p. 129-154.

FEIN, Elizabeth. 2011. Innocent machines: Asperger's syndrome and the neurostructural self. Sociological reflections on the neurosciences. Advances in medical sociology. Emerald Group Publishing, v. 13, p. 27-49.

FERREIRA, F. 2015. A negociação do diagnóstico de autismo. Monografia [Trabalho de Conclusão de Curso], Universidade Federal do Rio Grande do Sul, Porto Alegre.

FITZGERALD, Des. 2015. Autism, ignorance and love. Science and Culture, v. 22, n. 3, p. 394-400. FOUCAULT, Michel. 2006. O nascimento da clínica. Rio de Janeiro: Forense Universitária.

GIDDENS, Anthony. 1993. The Transformation of Intimacy. Sexuality, Love and Erotism in Modern Societies. Cambridge: Polity Press/Blackwell Publishers.

GINSBURG, Faye; RAPP, Rayna. 2013. Disability worlds. Annual Review of Anthropology, Palo Alto, v. 42, p. 53-68.

GRINKER, Roy Richard. 2010, Commentary: On being autistic, and social. Ethos: Journal of the Society for Psychological Anthropology, v. 38, issue 1, p. 172-178. Disponível em: https: / /anthrosource.onlinelibrary.wiley.com/doi/full/10.1111/j.1548-1352.2010.01087. Acesso em: 23 de maio de 2018.

GRINKER, Roy Richard; MANDEL, David. 2015. Notes on a puzzle piece. Autism, SAGE publications, p. 1-3. Disponível em: https://journals.sagepub.com/doi/full/10.1177/ 1362361315589293. Acesso em: 23 de maio de 2018.

HACKING, Ian. 2009. Autistic autobiography. The Philosophical Transactions of the Royal Society, n. 364, p. 1467-1473. Disponível em: https://royalsocietypublishing.org/doi/pdf/ 10.1098/ rstb.2008.0329. Acesso em: 23 de maio de 2018.

HACKING, Ian. 2007. Kinds of people. Moving targets. Proceedings of the British Academy, n. 151, p. 285-318.

HACKING, Ian. 2006. Making up people: clinical classifications. London Review of Books, v. $28, \mathrm{n}$. 16, August. Disponível em: https: / /www.lrb.co.uk/v28/n16/ian-hacking/making-up-people. 
Acesso em: 23 de maio de 2018.

HART, Brendan. 2014. Autism parents \& neurodiversity: radical translation, joint embodiment and the prosthetic environment. Biosocieties, v. 9, n. 3, p. 284-303.

HOLLIN, Gregory. 2014. Autism, sociality, and human nature. Somathosphere. Science, Medicine, and Anthropology. June, 18th. Disponível em: http: / / somatosphere.net/2014/06/autism-sociality-and-human-nature.html. Acesso em: 30 de maio de 2018.

HOLLIN, Gregory. 2014a. Construction a social subject:Autism and human sociality in the 1980s. History of the Human Sciences, v. 27, n. 4, p. 98-115.

JASANOFF, Sheila. 2004. The idiom of co-production. In: duction of science and social order. New York: Routledge. . States of knowledge: the copro-

LOPEZ, Rosa Maria Monteiro; SARTI, Cynthia. 2013. Eles vão ficando mais próximos do normal... Considerações sobre normalização na assistência ao autismo infantil. Ideias. Campinas, n. 6, nova série, 1, p. 77-98. Disponível em: https://periodicos.sbu.unicamp.br/ojs/index.php/ideias/article/ view/8649399. Acesso em: 10 de abril de 2017.

MALUF, Sônia W. 2015. Biolegitimacy, rights and social policies: New biopolitical regimes in mental healthcare in Brazil. Vibrant: Virtual Brazilian Anthropology, Brasília, v. 12, n. 1, June, p. 321-350.

MATTOS, Lisiane G de. 2016. Como se decide a (in)capacidade e a deficiência? Uma etnografia sobre moralidades e conflitos em torno da perícia médica previdenciária. Tese [Doutorado em Antropologia Social], Universidade Federal do Rio Grande do Sul, Porto Alegre.

MELLO, Anahi. G. de. 2016. "Deficiência, incapacidade e vulnerabilidade: do capacitismo ou a preeminência capacitista e biomédica do Comitê de Ética em Pesquisa da UFSC”. Ciência \& Saúde Coletiva, v. 21, n. 10, p. 3265-3276. Disponível em: http://www.scielo.br/scielo.php? pid=S1413-81232016001003265\&script $=$ sciabstract\&tlng=pt Acesso em: 10 de abril de 2017.

MELLO, Anahi Guedes; NUERNBERG, Adriano Henrique. 2012. Gênero e deficiência: intersecções e perspectivas. Estudos Feministas, Florianópolis, v. 20, n. 3, p. 635-655. Disponível em: https: / / periodicos.ufsc.br/index.php/ref/article/view/S0104-026X2012000300 003. Acesso em: $10 \mathrm{de}$ abril de 2017.

NUBILA, Heloisa Brunow Ventura Di. 2007. Aplicação das classificações CID-10 e CIF nas definições de deficiência e incapacidade. Tese [Doutorado em Saúde Pública], Universidade de São Paulo, São Paulo.

NUNES, Fernanda. 2014. Atuação política de grupos de pais de autistas no Rio de Janeiro: perspectivas para o campo da saúde. Dissertação [Mestrado em Saúde Coletiva], Instituto de Medicina Social, Rio de Janeiro.

OCHS, Elinor et al. 2004. Autism and the social world: an anthropological perspective. Discourse Studies, v. 6, n. 1, p. 147-183. London; Thousand Oaks; New Delhi: SAGE Publications.

ORTEGA, Francisco et al. 2013. A construção social do diagnóstico do autismo em uma rede social virtual brasileira. Interface. Comunicação, Saúde, Educação, v. 17, n. 44, jan/mar, p. 119-132. Disponível em: http://www.scielo.br/pdf/icse/v17n44/a10v17n44.pdf Acesso em: 10 de abril de 2017.

ORTEGA, Francisco. 2010. Deficiência, autismo e neurodiversidade. In: DINIZ, Débora \& SANTOS, Wederson (orgs.). Deficiência e Discriminação. Brasília: Letras e Livres. p. 143-168.

ORTEGA, Francisco. 2008. O sujeito cerebral e o movimento da neurodiversidade. Mana, v. 14, n. 2, p. 477-509. Disponível em: http://www.scielo.br/pdf/mana/v14n2/a08v14n2.pdf. Acesso em: 10 
de abril de 2017.

ORTEGA, Francisco. 2018. Commentary. Why not both? Negotiating ideias about autism in Italy, Brazil and the US. In: FEIN, Elizabeth; RIOS, Clarice (eds.). Autism in Translation. An Intercultural Conversation on Autism Spectrum Conditions. Culture, Mind and Society, University of Tulsa, USA. p. $89-108$.

ORTEGA, Francisco; CHOUDHURY, Suparna. 2011. 'Wired up differently: Autism, adolescence and the politics of neurological identities. Subjetivity. Macmillan Publishers, v. 4, n. 3, p. 323-345.

RABINOW, Paul. 2008. Afterword: concept work. In: GIBBON, Sahra; NOVAS, Carlos. Biosocialities, genetics and the social sciences: making biologies and identities, Taylor \& Francis e-Library.

RIOS, Clarice; ORTEGA, Francisco; ZORZANELLI, Rafaela; NASCIMENTO, Leonardo Fernandes. 2015. Da invisibilidade à epidemia. A construção narrativa do autismo na mídia impressa brasileira. Interface: Comunicação, Saúde, Educação. Rio de Janeiro, v. 19, n. 53, p. 325-336. Disponível em: http://www.scielo.br/pdf/icse/v19n53/1807-5762-icse-1807-576220140146. pdf. Acesso em: 10 de abril de 2017.

RIOS, Clarice. 2018. Experts on your own child, expert on your own world. Reinventing autism expertise(s). In: FEIN, Elizabeth; RIOS, Clarice (eds.). Autism in Translation. An Intercultural Conversation on Autism Spectrum Conditions. Culture, Mind and Society, University of Tulsa, USA, p. 185-206.

RIOS, Clarice; ANDRADA, Bárbara Costa. 2015. The changing face of autism in Brazil. Culture, Medicine and Psychiatry, v. 39, issue 2, April, p. 213-234. Disponível em: https: / / leicufrj.files. wordpress.com/2017/05/rios-clarice-the-changing-face-of-autism1.pdf. Acesso em: 10 de abril de 2017.

ROSE, Nikolas. 1997. Assembling the modern self. In: PORTER, Roy (ed.). Rewriting the self. Histories from the Renaissance to the present. London; New York: Routledge, p. 224-288.

ROSE, Nikolas. 2008. Psicologia como uma ciência social. Psicologia e Sociedade, v. 20, n. 2, p. $155-$ 164. Disponível em: http://www.scielo.br/pdf/psoc/v20n2/a02v20n2.pdf. Acesso em: 23 de maio de 2018.

ROSE, Nikolas. 2013. The human sciences in a Biological Age. Theory, Culture and Society, v. 30, p. 1, p. 3-34.

ROSENBERG, Charles E. 2002. The tyranny of diagnosis: Specific entities and individual experience. The Milbank Quarterly, v. 80, n. 2, p. 237-260. Disponível em: https://www.ncbi.nlm.nih.gov/pmc/ articles/PMC2690110/. Acesso em: 23 de maio de 2018.

SILVERMAN, Chloe. 2008. Fieldwork in another planet: Social Science Perspectives on the Autism Spectrum. Biosocieties, v. 3, issue 03, Sept., p. 325-341, Cambridge: Cambridge Journals.

SOLOMON, Olga. 2013. Autism affordances of achievement. Narrative genres and parenting practices. In: LONG, Nicholas J.; MOORE, Henrietta L. The social life of achievement. New York; Oxford: Berghahn. p. 120-138.

VALTELLINA, Enrico. 2018. A. S. Classification, Interpellation. In: FEIN, Elizabeth; RIOS, Clarice (eds.). Autism in Translation. An Intercultural Conversation on Autism Spectrum Conditions. Culture, Mind and Society, University of Tulsa, USA, p. 207-230. 
$A$ (des)construção social do diagnóstico de autismo no contexto das políticas de cotas para pessoas com deficiência no mercado de trabalho

Resumo: No cenário de uma crescente propagação midiática sobre o autismo, a flexibilidade e a complexidade da sua produção como um “diagnóstico clínico" e situado em um espectro altamente diversificado, somam-se aos debates sobre esta condição ser uma deficiência ou uma neurodiversidade uma “maneira de ser". A partir de uma etnografia que contou com entrevistas em profundidade com neurologistas e psiquiatras; uma convivência de três anos com dois jovens diagnosticados com autismo em seus espaços de trabalho e sociabilidade; e o acompanhamento do cotidiano de trabalho de assessoria em inclusão de uma especialista no assunto, analiso a construção social do diagnóstico do autismo e os efeitos das diferentes formas de entender, "habitar" e agenciar esta condição nos espaços laborais. Tal pesquisa permitiu-me problematizar a centralidade do conhecimento neurobiológico e dos especialistas psi e relativizar estes saberes na produção diagnóstica do autismo e na efetivação da política de cotas. Analiso também as estratégias de inserção do autismo na agenda pública, mostrando, na prática, como os diversos atores envolvidos nas disputas em torno do seu status nosológico coproduzem novos sujeitos, relações e sensibilidades sociais no Brasil.

Palavras-chave: Autismo, Deficiência, Processos Diagnósticos, Trabalho, Políticas Públicas.
The social (de)construction of the autism diagnosis in the context of quota policies for people with disabilities in the labour market

Abstract: In the context of a growing representation of autism in the media, the flexibility and complexity of its production as a "clinical diagnosis" and the fact that it is situated in a highly diversified spectrum, add to debates about this condition being a disability or neurodiversity - a "way of being”. Through an ethnographic work that ranged from in-depth interviews with neurologists and psychiatrists; a participant-observation of three years with two young people diagnosed with autism in their labour routines and spaces of sociability; and the follow-up of the day-by-day work of an expert in autism, I analyse the social construction of the autism diagnosis and the effects of different modes of understanding, "inhabiting" and agenting this condition in the workplace. My research allowed me to both problematize the centrality of neurobiological knowledge and psi specialists, and to relativize these knowledges both within the production of the autism diagnostic and the implementation of the quota policy. I also discussed the strategies of insertion of autism in the public agenda, showing, in practice, how the various actors involved in the disputes around autism's nosological status co-produce new subjects, relations and social sensibilities in Brazil.

Keywords: Autism, Disability, Diagnostic Processes, Work, Public Policies. 